\title{
Macrolide Resistant Mycoplasma pneumoniae in Community Acquired Pneumonia
}

\author{
Serena M. Varghese ${ }^{1} \cdot$ Vishakha V. Kerkar ${ }^{1}$ \\ Received: 28 January 2020 / Accepted: 8 April 2020 / Published online: 24 April 2020 \\ (C) Dr. K C Chaudhuri Foundation 2020
}

To the Editor: Mycoplasma pneumoniae (MP) is a major cause of community acquired pneumonia (CAP) in children. Macrolides are the first-choice antimicrobials for CAP due to MP in children but increasing reports of macrolide resistance from other countries are of concern [1]. A healthy 12-y-old presented with one week history of high fever and cough. Evaluation showed features of right lower lobe pneumonia with leukopenia, elevated inflammatory markers and positive mycoplasma immunoglobulin M. He was started on ceftriaxone, fluvir and azithromycin. As he continued to be febrile with rising inflammatory markers and worsening X- ray, antibiotics were upgraded and a CT scan was taken which showed right lower lobe consolidation with an accessory cardiac bronchus which was not communicating with the area of consolidation. Bronchoalveolar lavage (BAL) culture was negative but real time polymerase chain reaction (PCR) test was positive for MP. Molecular study by PCR detected erythromycin ribosome methylation (Erm) genes, Erm B and Erm $F$ responsible for macrolide resistance. So IV antibiotics were stopped and Tab. doxycycline was administered for two weeks. Left sided minimal pneumothorax which developed after bronchoscopy improved with symptomatic treatment.

The first case of macrolide resistant MP (MR MP) was reported in a child in Japan in 2001 [2]. A major mechanism of resistance to macrolides is mediated by Erm genes that encode rRNA methyltransferases [3]. Ki Bae Hong et al. attributed an increasing prevalence of MR MP in South Korea to increased use of macrolides, high population density and geographic closeness to countries with high macrolide resistance [1]. Macrolides were included in the watch category of the 2017 revision of the WHO model list of essential

Serena M. Varghese

serena.mohan@ rajagirihospital.com

1 Department of Pediatrics, Rajagiri Hospital, Aluva, Kochi 683112, India medicines, which signifies higher potential to develop resistance [4]. A study done in 2011 in India showed an increasing sale of antibiotics including macrolides [5].

To the best of our knowledge, the present report is the first documented case of MR MP strain from India. In the context of increasing use of macrolides in India, we suggest that MR MP should be considered as a differential diagnosis when one encounters cases of deteriorating CAP.

\section{Compliance with Ethical Standards}

Conflict of Interest None.

\section{References}

1. Hong KB, Choi EH, Lee HJ, et al. Macrolide resistance of mycoplasma pneumoniae, South Korea, 2000-2011. Emerg Infect Dis. 2013;19:1281-4.

2. Okazaki N, Narita M, Yamada S, et al. Characteristics of macrolideresistant mycoplasma pneumoniae strains isolated from patients and induced with erythromycin in vitro. Microbiol Immunol. 2001;45: 617-20.

3. Fyfe C, Grossman TH, Kerstein K, Sutcliffe J. Resistance to macrolide antibiotics in public health pathogens. Cold Spring Harb Perspect Med. 2016;6:a025395.

4. WHO. Executive summary: The Selection and Use of Essential Medicines. Report of the 21 st WHO expert committee on the selection and use of essential medicines. Available at: http://www.who.int/ medicines/publications/essential medicines/EML_2017 ExecutiveSummary.pdf. Accessed September 25, 2019.

5. Ganguly NK, Arora NK, Chandy SJ, et al. Rationalizing antibiotic use to limit antibiotic resistance in India. Indian J Med Res. 2011;134:281-94.

Publisher's Note Springer Nature remains neutral with regard to jurisdictional claims in published maps and institutional affiliations. 